\title{
Parámetros de tratamiento por aire caliente en la conservación del aguaymanto (Physalis peruviana) en dos estados de madurez
}

\section{Parameters of hot air treatment on the conservation of golden berry (Physalis peruviana) at two maturity stages}

\author{
Miriam E. Ramos ${ }^{1, a, *}$, Ana M. Matos ${ }^{2, b}$, Nicolás M. Espinoza ${ }^{1, c}$, Oscar B. Jordán ${ }^{3, d}$ \\ ${ }^{1}$ Escuela Profesional de Ingeniería Agroindustrial, Facultad de Ciencias Agrarias, Universidad Nacional Hemilio \\ Valdizan, Huánuco, Perú. \\ 2 Escuela Profesional de ingenieria Civil, Facultad de Ingeniería Civil y Arquitectura, Universidad Nacional Hemilio \\ Valdizan, Huánuco, Perú. \\ ${ }^{3}$ Facultad de Ciencia de los Alimentos, Universidad Le Cordon Bleu, Lima, Perú. \\ a Ph.D., miriamramos@unheval.edu.pe, (1D https://orcid.org/0000-0002-3970-2857 \\ b Dra., amatos@unheval.edu.pe, (D) https://orcid.org/0000-0002-0452-5037 \\ c Ing., 囚 merlinespinozablas@gmail.com, (D) https://orcid.org/0000-0003-4350-1836 \\ d Ph.D., Q $_{\text {oscar.jordan@ulcb.edu.pe, (10 https://orcid.org/0000-0002-1280-7704 }}$ \\ * Autor de Correspondencia: Tel. +51 958578152 \\ http://dx.doi.org/10.25127/riagrop.20212.677
}

http://revistas.untrm.edu.pe/index.php/RIAGROP revista.riagrop@untrm.edu.pe

Recepción: 26 de enero 2021

Aprobación: 27 de marzo 2021

Este trabajo tiene licencia de Creative Commons. Attribution-NonCommercial-ShareAlike $\quad 4.0$ International Public License - CC-BY-NC-SA 4.0

\section{Resumen}

Se estudió el efecto del tratamiento por aire caliente a 40 y $46^{\circ} \mathrm{C}$ por 40,50 y $60 \mathrm{~min}$. y un testigo, en la conservación poscosecha del aguaymanto en el estado de madurez 4 y 5 con cáliz; almacenados a $4 \pm 0.5^{\circ} \mathrm{C}$. Se evaluaron los siguientes atributos de calidad: Color, textura, $\mathrm{pH}, \%$ Acidez expresado en ácido cítrico y sólidos solubles y se tomaron muestras cada 15 días por 30 días. El análisis comparativo de diferencia estadística, por atributo entre los estados de madurez 4 y 5, arrojó diferencias para los atributos $\mathrm{pH}$ y textura y color, a los 0 y 15 días de almacenamiento, respectivamente. Estos atributos fueron comparados con el testigo y se encontró menor diferencia significativa con el estado de madurez 5. Sin embargo, al optimizar los factores $(\mathrm{pH}$, textura y color) se 
evidenció que el mejor tratamiento consiste en $46^{\circ} \mathrm{C}$ de temperatura, un tiempo de $54 \pm 6$ min y un estado de madurez de 4. El aguaymanto obtenido bajo esta modalidad presentó las siguientes caracteristicas: Sólidos solubles $15.17^{\circ}$ Brix, $\mathrm{pH}=3.95$; \% Acidez exp en ácido cítrico 1.93, Coordenadas de color $\mathrm{L}^{*}=65.05, \mathrm{a}^{*}=14.77$, $\mathrm{b}^{*}=52.80$ y textura $\mathrm{gf} 230.90$.

Palabras Clave: Aguaymanto, estado de madurez, textura, poscosecha, color.

\begin{abstract}
Golden berries with calyx treated at 40 and $60^{\circ} \mathrm{C}$ for 40,50 and 60 minutes at two degrees of ripeness were studied during 30 days of storage at $4.0 \pm 0.5^{\circ} \mathrm{C}$ ). Several physicochemical characteristics (Color, Texture, $\mathrm{pH}$, acidity and soluble solids) were determined for each sample at 0,15 and 30 days. There were significant differences among degrees of ripeness 4 and 5 for $\mathrm{pH}$ and texture at 0 days of storage, and for color after 15 days. These properties were compared to a standard sample (with no heat treatment), and the degree of ripeness number 5 was very similar to the standard, while stage 4 had higher difference. In order to optimize the levels of the factors significant (Temperature, Time and Degree of ripeness), these tree factors were analyzed using Response Surface Method and it was found that the formula which optimizes Texture, $\mathrm{pH}$ and Color is: $46^{\circ} \mathrm{C}$ for Temperature, $54 \pm 6 \mathrm{~min}$ of time, and Degree of ripeness number 4 . Samples treated under optimize conditions were characterized as follows: soluble solids $=15.17^{\circ} \mathrm{Brix}, \mathrm{pH}=3.95$, acidity $=1.93$, color $\left(\mathrm{b}^{*}\right.$, CIELab): 52.80 and texture=230.0gf.
\end{abstract}

Keywords: golden berry, degree of ripeness, texture, postharvest, color.

\section{INTRODUCCIÓN}

La uchuva (Physalis peruviana L.) es una fruta que ha cobrado importancia en los mercados internacionales, principalmente la Unión Europea. Es valorada por su calidad organoléptica $\mathrm{y}$, recientemente, por sus propiedades anticancerígenas (Quintero et al., 2012). En la actualidad se registran pérdidas de poscosecha bastantes significativas que superan el $20 \%$ dentro del sector hortofrutícola, destacando las frutas nutricionalmente por el aporte de energía, fibra, minerales y vitaminas principalmente el ácido ascórbico, complejo B y Provitaminas A, pigmentos que se encuentran como sustancias antioxidantes (Rolz, 2011).

La presente investigación se plantea para la conservación de frutos andinos tipo baya (Aguaymanto) y su acondicionamiento antes de su almacenamiento en frío, con la aplicación de tratamiento con aire caliente para eliminar la carga microbiana superficial y la presencia de insectos $u$ otros vectores biológicos que afectan la calidad de la fruta (Rolz, 2011). Asimismo, el efecto del tratamiento por calor induce una resistencia a los daños por frío, retraso en la maduración y senescencia (Quintero et al., 2012). Al respecto, Ramos (2011) señala que mediante los tratamientos por calor se consigue disminuir la velocidad de respiración del fruto y liberación del etileno; evitar pérdidas de firmeza al afectar a enzimas asociadas a la degradación de la pared celular y desórdenes fisiológicos pronunciados, a causa de las bajas temperaturas y senescencias y reducir, e incluso eliminar, el ataque de ciertos microorganismos patógenos. Todos estos criterios conducen a un estudio de aplicación y 
determinar parámetros de control en la conservación de estos frutos que en nuestra región constituyen un potencial por las condiciones climáticas propias para su cultivo, que permita incrementar su comercialización bajo los estándares exigidos por el mercado nacional e internacional sin pérdidas considerables en su manejo.

En función a estos aspectos se plantearon los siguientes objetivos: evaluar el estado de madurez en la calidad y vida útil de aguaymanto (Physalis peruviana) en refrigeración y determinar el efecto del tratamiento por aire caliente en la calidad y vida útil de aguaymanto (Physalis peruviana).

\section{MATERIALES Y MÉTODOS}

El trabajo de investigación se realizó en las instalaciones de los Laboratorios de la Universidad Nacional Hermilio Valdizán, en la Facultad de Ciencias Agrarias y Escuela Profesional de Ingeniería Agroindustrial (EPIA), también se realizó en los Campos de Cultivo de Huandobamba-Ambo, de la empresa Bayas Peruanas E.I.R.L.

\subsection{Materia prima y reactivos}

Se utilizó aguaymanto en los estados de madurez del 2 al 6 recolectados de la finca Bayas Peruanas E.I.R.L. durante los meses de abril a junio, procedente de la localidad de Ambo.

Los reactivos que se emplearon fueron: hidróxido de sodio $(\mathrm{NaOH})$, fenolftaleína, DNS (ácido-3,5-dinitrosalicílico), ácido ascórbico, oxálico, caroteno, acetona y hexano.

\subsection{Métodos de análisis físico-químicos}

2.2.1. Características biométricas de los frutos: Se registró longitud, diámetro y peso de las bayas de acuerdo a las recomendaciones de Ramos (2011).

2.2.2. $p H$ y sólidos solubles: Por el método A.O.A.C (1997).

2.2.3. Acidez: Basado en la técnica volumétrica de Pearson (Ramos, 2011). El resultado se expresó en porcentaje de ácido cítrico (AA).

2.2.4. Color: Medición de coordenadas de color según CIE L*, $\mathrm{a}^{*}, \mathrm{~b}^{*}$.

2.2.5. Textura: Por el método recomendado por Rolle et al. (2012).

2.2.6. Carotenoides: Por el método descrito por Talcott y Howard (1999).

2.2.7. Vitamina $\quad$ C: $\quad$ Se determinó cuantitativamente por el método espectrofotométrico basado en la reducción del colorante 2,6 diclorofenol indofenol por una solución de ácido (Ramos, 2011).

\subsection{Evaluación sensorial}

Se realizaron pruebas de preferencia y comparación pareada a través de un panel conformado por 21 jueces semi-entrenados de la EPIA a fin de seleccionar los estados de madurez apropiados para el estudio (Ramos, 2013).

\subsection{Tratamientos en estudio}

El estudio comprendió dos etapas. En la primera etapa se evaluaron 12 combinaciones de factores de temperatura $\left(40\right.$ y $\left.46{ }^{\circ} \mathrm{C}\right)$ y tiempo (40, 50 y $60 \mathrm{~min})$, teniendo como variable respuesta la textura, seleccionando al mejor tratamiento de aguaymanto con cáliz o sin él, los que fueron analizados estadísticamente mediante un diseño de bloques con arreglo 
factorial $(2 * 3)$ y la prueba de comparación Tukey con $\alpha=5 \%$.

En la segunda etapa, se muestran los tratamientos estudiados al material vegetal (tabla 1) para elegir al mejor tratamiento por aire caliente, que permita extender la vida útil del producto. Esta operación fue previa al almacenamiento en refrigeración a $4{ }^{\circ} \mathrm{C}$ aproximadamente.

Los tratamientos en estudio fueron estadísticamente analizados mediante un Diseño Multi Nivel Factorial. Los bloques fueron constituidos por cada estado de madurez y se tuvo como factores la temperatura (dos niveles) y el tiempo (tres niveles), como se detalla:

Boque : Representa dos estados de madurez (4 y 5).

Factor 1 : Las dos temperaturas del tratamiento por aire caliente $\left(40\right.$ y $\left.46^{\circ} \mathrm{C}\right)$.

Factor 2: Los tres tiempos del tratamiento por aire caliente (40, 50 y $60 \mathrm{~min}$.).

Tabla 1. Tratamientos ( $T$ ) por estados de madurez 4 y 5 , a diferentes temperaturas (Temp) y tiempo $(t)$

\begin{tabular}{cccc}
\hline $\mathbf{T}$ & Bloque & $\begin{array}{r}\text { Factor 1: } \\
\text { Temp }\left({ }^{\circ} \mathbf{C}\right)\end{array}$ & $\begin{array}{c}\text { Factor 2: } \\
\mathbf{t}(\mathbf{m i n})\end{array}$ \\
\hline 1 & 1 & 40 & 40 \\
2 & 1 & 46 & 40 \\
3 & 1 & 40 & 50 \\
4 & 1 & 46 & 50 \\
5 & 1 & 40 & 60 \\
6 & 1 & 46 & 60 \\
7 & 2 & 40 & 40 \\
8 & 2 & 46 & 40 \\
9 & 2 & 40 & 50 \\
10 & 2 & 46 & 50 \\
11 & 2 & 40 & 60 \\
12 & 2 & 46 & 60 \\
\hline $1: E$
\end{tabular}

1: Estado de madurez 4 2: Estado de madurez 5
Cuando se identificaron los atributos significativamente diferentes entre 4 y 5 , se realizó un diseño Multi Nivel Factorial y se emplearon tres variables (Tiempo, Temperatura, y Estado de Madurez) a 3, 2 y 2 niveles, respectivamente, para la determinación del mejor tratamiento. El diseño empleado se muestra en la tabla 2.

Tabla 2. Tratamiento (T) por estados de madurez 4 y 5 , a diferentes temperaturas (Temp) y tiempo $(t)$

\begin{tabular}{cccc}
\hline $\mathbf{T}$ & $\mathbf{t}(\mathbf{m i n})$ & $\begin{array}{c}\text { Temp } \\
\left({ }^{\circ} \mathbf{C}\right)\end{array}$ & $\begin{array}{c}\text { Estado de } \\
\text { madurez }\end{array}$ \\
\hline 1 & 40 & 40 & 4 \\
2 & 50 & 40 & 4 \\
3 & 60 & 40 & 4 \\
4 & 40 & 46 & 4 \\
5 & 50 & 46 & 4 \\
6 & 60 & 46 & 4 \\
7 & 40 & 40 & 5 \\
8 & 50 & 40 & 5 \\
9 & 60 & 40 & 5 \\
10 & 40 & 46 & 5 \\
11 & 50 & 46 & 5 \\
12 & 60 & 46 & 5 \\
\hline
\end{tabular}

\subsection{Metodología experimental}

\subsubsection{Caracterización del aguaymanto}

El estudio comprendió la evaluación del aguaymanto comercializado en el centro de acopio, en su madurez comercial, de dos altitudes de procedencia: Huandobamba a 2400 m s. n. m. e Ichocan a 2800 m s. n. m. Este fue caracterizado en peso, diámetro ecuatorial, longitud, sólidos solubles, $\mathrm{pH}$, acidez, color, textura y evaluados en porcentaje de pérdidas de peso del aguaymanto con cáliz y sin cáliz almacenados a condiciones ambientales durante 9 días. 
2.5.2. Estudio del aguaymanto por estados de madurez

Se realizó la recolección de los estados de madurez del 2 al 6, que fueron caracterizados en peso, longitud, diámetro ecuatorial, sólidos solubles, $\mathrm{pH}$, acidez, color $\mathrm{y}$ textura. Asimismo, estos estados de madurez fueron almacenados bajo condiciones ambientales y evaluados después de 13 días de almacenamiento a través de la prueba de preferencia por un panel conformado por 21 jueces semientrenados de la EPIA. Esta evaluación permitió identificar los estados de madurez más preferidos para la caracterización en vitamina $\mathrm{C}$, carotenoides y la aplicación del tratamiento por calor $y$ almacenamiento en refrigeración.

2.5.3. Estudio de la textura del aguaymanto con cáliz y sin él en almacenamiento postratamiento por aire caliente

Se evaluó el aguaymanto en el estado de madurez 4, previo al tratamiento por aire caliente monitoreando la textura a los 0 y 15 días durante almacenamiento para tomar decisiones y continuar con la investigación.

\subsubsection{Evaluación de características de calidad en almacenamiento del aguaymanto postratamiento por aire caliente}

En la figura 1, se muestra el esquema de trabajo que comprendió inicialmente los controles por estados de madurez 4 y 5 para la caracterización fisicoquímica de los frutos y los respectivos controles a los 0,15 y 30 días, a condiciones de refrigeración de los indicadores de calidad: color, textura, sólidos solubles ( ${ }^{\circ}$ Brix), acidez y $\mathrm{pH}$.

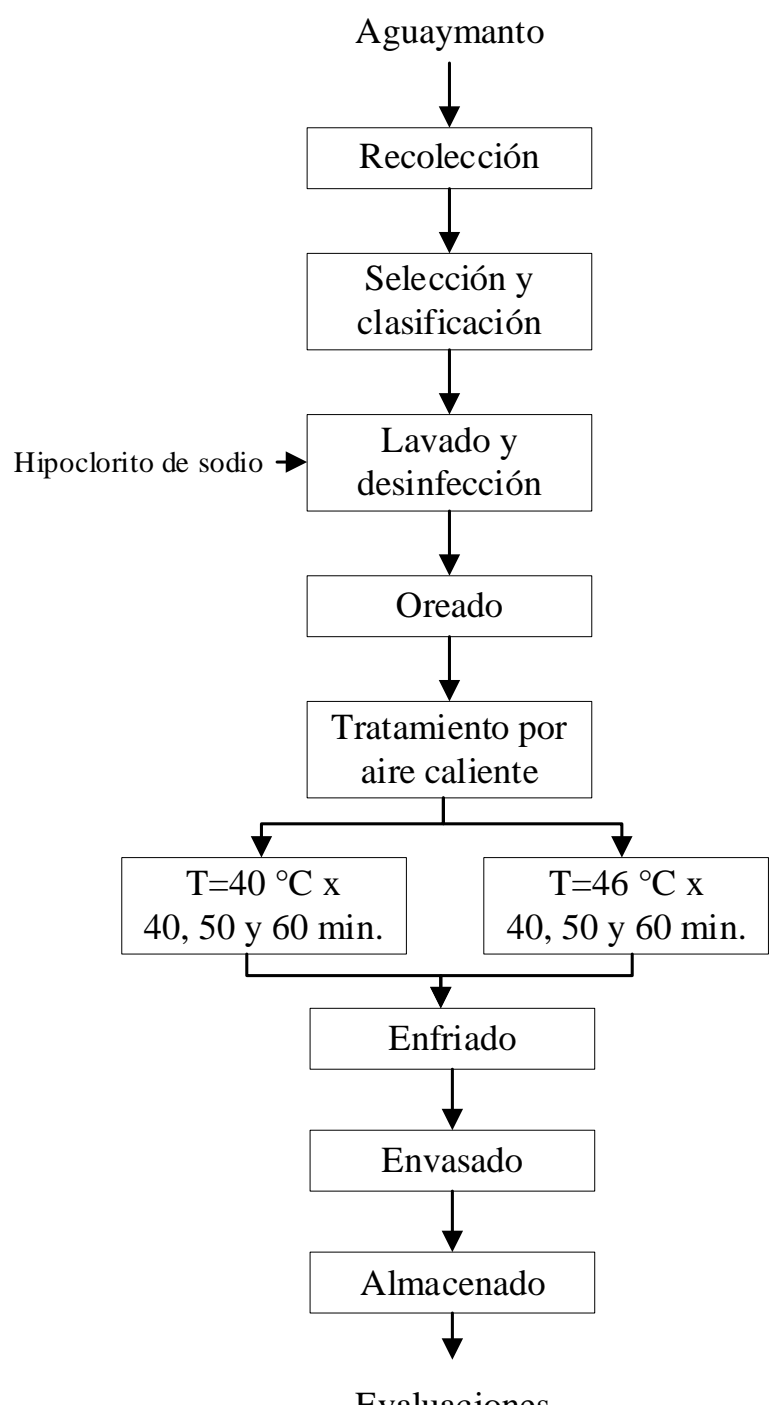

Figura 1. Diagrama de bloques del manejo poscosecha.

\section{RESULTADOS}

\subsection{Caracterización \\ fisicoquímica \\ del aguaymanto}

En la tabla 3, se muestra los atributos de calidad del aguaymanto recolectado del centro de acopio de dos altitudes de procedencia. 
Tabla 3. Resultados promedios de la caracterización del aguaymanto procedente de dos altitudes

\begin{tabular}{|c|c|c|}
\hline Procedencia & Características & Resultados \\
\hline \multirow{12}{*}{ Huandobamba } & Peso $(\mathrm{g})$ & 3.76 \\
\hline & Longitud (mm) & 18.30 \\
\hline & Diámetro ecuatorial (mm) & 18.45 \\
\hline & Sólidos solubles ( ${ }^{\circ}$ Brix) & 15.37 \\
\hline & $\mathrm{pH}$ & 3.46 \\
\hline & \% Acidez expresado en ácido cítrico & 2.10 \\
\hline & Color: & \\
\hline & $\mathrm{L}^{*}$ & 66.8 \\
\hline & $a^{*}$ & 21.5 \\
\hline & $b^{*}$ & 53.8 \\
\hline & $\mathrm{C}^{*}$ & 58 \\
\hline & $\mathrm{h}^{*}$ & 68.2 \\
\hline \multirow{12}{*}{ Ichocan } & Peso $(g)$ & 5.25 \\
\hline & Longitud (mm) & 20.03 \\
\hline & Diámetro ecuatorial $(\mathrm{mm})$ & 20.40 \\
\hline & Sólidos solubles ( ${ }^{\circ}$ Brix $)$ & 14.50 \\
\hline & $\mathrm{pH}$ & 3.59 \\
\hline & \%Acidez expresado en ácido cítrico & 2.14 \\
\hline & Color: & \\
\hline & $\mathrm{L}^{*}$ & 62.3 \\
\hline & $a^{*}$ & 21.7 \\
\hline & $b^{*}$ & 54.1 \\
\hline & $C^{*}$ & 58.3 \\
\hline & $h^{*}$ & 68.1 \\
\hline
\end{tabular}

En la tabla 4, se muestra el estudio de pérdidas de peso a condiciones ambientales del aguaymanto procedente de dos altitudes, con cáliz y sin cáliz, después de 9 días de almacenamiento.

\subsection{Estudio del aguaymanto por estados de madurez}

En la tabla 5, se muestra la caracterización de cinco estados de madurez en este fruto de acuerdo a los criterios recomendados por la norma técnica colombiana.

Tabla 4. Resultados de la evaluación del porcentaje de pérdidas de peso

\begin{tabular}{clc}
\hline \multicolumn{1}{c}{ Procedencia } & \multicolumn{1}{c}{ Características } & Resultados \\
\hline & Pérdida de peso (\%): & 16.70 \\
& Aguaymanto con cáliz & 21.10 \\
& Aguaymanto sin cáliz & 231.23 \\
\hline & Texturagf* & \\
Ichocan & Pérdida de peso (\%): & 17.64 \\
& Aguaymanto con cáliz & 19.44 \\
& Aguaymanto sin cáliz & 249.18 \\
\hline
\end{tabular}

Nota: * Almacenadas a condiciones de refrigeración con cáliz 
Tabla 5. Resultados promedios de la caracterización del aguaymanto en 5 estados de madurez

\begin{tabular}{lrrrrr}
\hline \multirow{2}{*}{ Características } & \multicolumn{6}{c}{ Estados de madurez } \\
\cline { 2 - 6 } & \multicolumn{1}{c}{$\mathbf{2}$} & \multicolumn{1}{c}{$\mathbf{3}$} & \multicolumn{1}{c}{$\mathbf{5}$} & $\mathbf{6}$ \\
\hline Peso $(\mathrm{g})$ & 2.6 & 3.46 & 3.49 & 3.93 & 4.21 \\
Longitud (mm) & 16.8 & 17.49 & 18.07 & 12.72 & 12.55 \\
Diámetro ecuatorial $(\mathrm{mm})$ & 16.32 & 16.67 & 17.11 & 12.21 & 11.91 \\
Sólidos solubles ( ${ }^{\circ}$ Brix) & 12.2 & 14.07 & 14.7 & 14.67 & 14.97 \\
pH & 3.67 & 3.71 & 3.72 & 3.71 & 3.78 \\
\%Acidez exp. en AA & 2.33 & 2.31 & 2.16 & 2.1 & 1.81 \\
Color** & & & & & \\
L $^{*}$ & 70.19 & 67.36 & 67.91 & 67.47 & 66.1 \\
$\mathrm{a}^{*}$ & 10.7 & 14.08 & 15.15 & 14.57 & 15.22 \\
$\mathrm{~b}^{*}$ & 54,27 & 53.23 & 54.89 & 53.4 & 54.41 \\
Texturagf* & 244.5 & 212.4 & 189.5 & 172.8 & 156.4 \\
\hline
\end{tabular}

Nota: ${ }^{* *}$ Reportes a los 12 días de almacenamiento a condiciones ambientales.

Para decidir por los estados de madurez a trabajar en el manejo poscosecha del aguaymanto, los frutos almacenados a condiciones ambientales por 13 días se sometieron a una evaluación sensorial denominada "Prueba de preferencia". Los estados de madurez recolectados $\mathrm{y}$ codificados para este análisis fueron: 2 (280), 3 (295), 4 (236), 5 (250). No se seleccionó el estado de madurez 6, por encontrarse en su madurez comercial y estar próximo a la senescencia. Los resultados se muestran a continuación. Se observó diferencias significativas entre los estados de madurez 2 y 4. Por el contrario, los estados de madurez 3 y 5 no presentaron diferencias significativas.

Por otro lado, en la tabla 6, se registra el contenido de Vitamina C y carotenoides, estos componentes fueron cuantificados para los estados de madurez 4 y 5, muestras de mayor preferencia por el panel de evaluación sensorial.

Tabla 6. Resultados de la determinación de vitamina $C$ y carotenoides en los estados de madurez 4 y 5

\begin{tabular}{clc}
\hline $\begin{array}{c}\text { Estados de } \\
\text { madurez }\end{array}$ & \multicolumn{1}{c}{ Características } & Resultados \\
\hline \multirow{2}{*}{4} & Vitamina C mg/100g & 28.21 \\
& mg equivalente de $\beta$ - Caroteno/100 g & 3.04 \\
\hline \multirow{2}{*}{5} & Vitamina C mg/100g & 25.83 \\
& mg equivalente de $\beta$ - Caroteno/100 g & 4.23 \\
\hline
\end{tabular}




\subsection{Estudio de la textura del aguaymanto con cáliz y sin él en almacenamiento post- tratamiento por aire caliente}

En la figura 2, se muestra los promedios de textura del estado de madurez 4 sometido al tratamiento por aire caliente $\mathrm{y}$ evaluado durante almacenamiento en refrigeración $4{ }^{\circ} \mathrm{C}$ al cabo de 0 y 15 días.

\subsection{Evaluación de características de calidad en almacenamiento del aguaymanto post- tratamiento por aire caliente}

En la tabla 7, se presenta un resumen de las diferencias significativas encontradas al evaluar cada atributo de calidad entre los estados de madurez 4 y 5, a los 0, 15 y 30 días de almacenamiento, respectivamente.

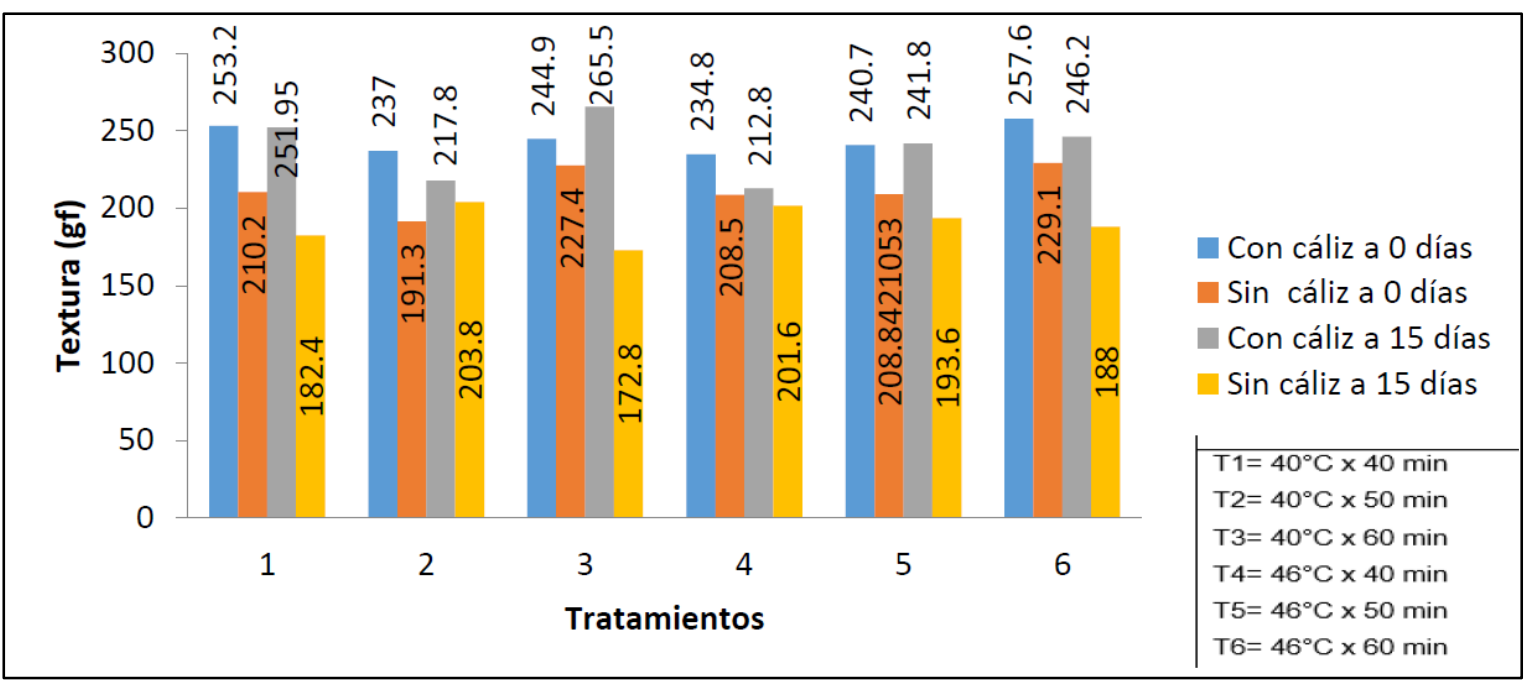

Figura 2. Resultados promedios de textura del estado de madurez 4 en almacenaje.

Tabla 7. Análisis comparativo de diferencia estadística por atributo entre estados de madurez 4 y 5

\begin{tabular}{lccc}
\hline \multirow{2}{*}{\multicolumn{1}{c}{ Indicador }} & \multicolumn{3}{c}{ Tiempo (días) } \\
\cline { 2 - 4 } & $\mathbf{0}$ & $\mathbf{1 5}$ & $\mathbf{3 0}$ \\
\hline Acidez & N.S. & N.S. & N.S. \\
Sólidos Solubles $\left({ }^{\circ}\right.$ Brix $)$ & N.S. & N.S. & N.S. \\
$\mathrm{pH}$ & S. & N.S. & N.S. \\
Textura $\left(\mathrm{g}_{\mathrm{f}}\right)$ & S. & N.S. & N.S. \\
Color $\left(\mathrm{b}^{*}\right)$ & N.S. & S. & N.S. \\
\hline
\end{tabular}

${ }^{*}$ N.S.: No significativo. ${ }^{*}$ S.: Significativo

En comparación al testigo, a los 0 días se observa que para el $\mathrm{pH}$ existe diferencia significativa con las muestras de estado de madurez 4. Sin embargo, no existe diferencia con las muestras en estado de madurez 5; por tanto, el mejor estado de madurez sería el 5. En cuanto a la textura, a los 0 días no existe diferencia entre el testigo y el estado de madurez 4. Fue este mejor en comparación al 5, porque sí muestra diferencia significativa con el testigo. 
A los 15 días, la variación del color resultó significativa, en comparación con el testigo. Además, se observó que el mejor estado de madurez es el 5, ya que no presenta variación con el testigo.

Se evaluó el tratamiento óptimo para maximizar el $\mathrm{pH}$ (sin incluir el testigo) mediante un diseño multifactorial a los 0 días y se encontró que existe una zona en donde la combinación de los niveles factores (Estado de madurez, tiempo y temperatura) maximizan el $\mathrm{pH}$, como se muestra en la figura 3 .
Asimismo, para la textura (a los 0 días), se encontró que si bien no existe un área claramente definida, se evidencia una zona de mayor valor para la textura, como se aprecia en la figura 4.

El atributo color, que resultó significativo a los 15 días se muestra en la figura 5.

Al analizar los tres atributos de calidad que resultaron significativos, el $\mathrm{pH}$ y la textura (0 días), y color (15 días), los niveles óptimos se muestran en la tabla 8.

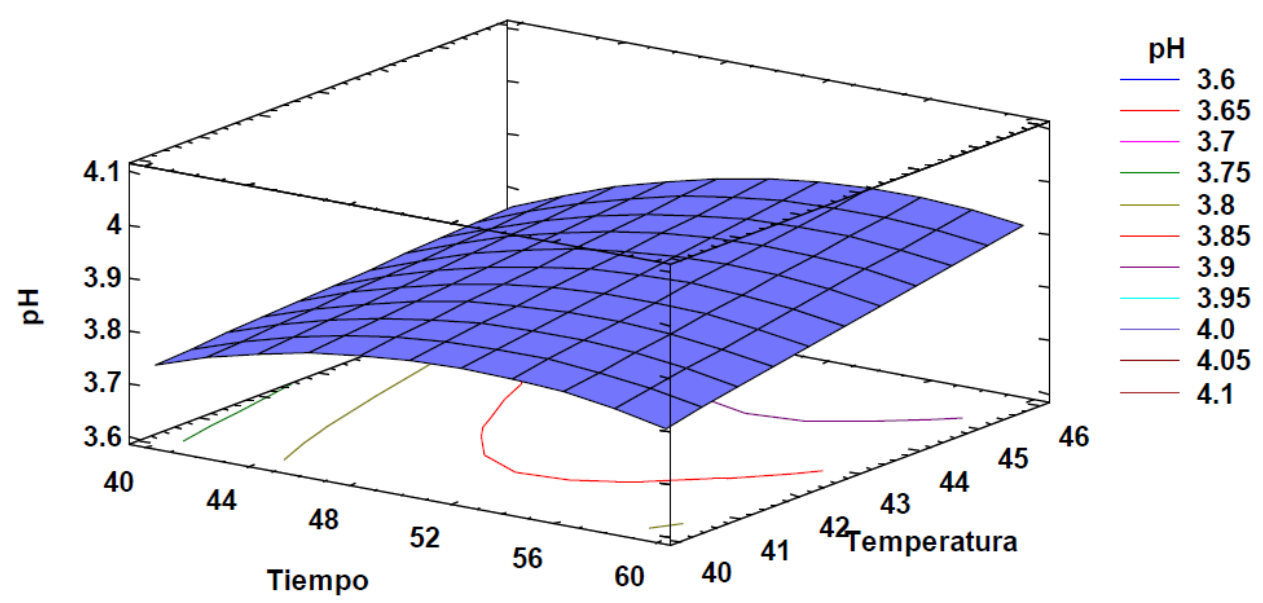

Figura 3. Superficie de respuesta del pH a los 0 días.

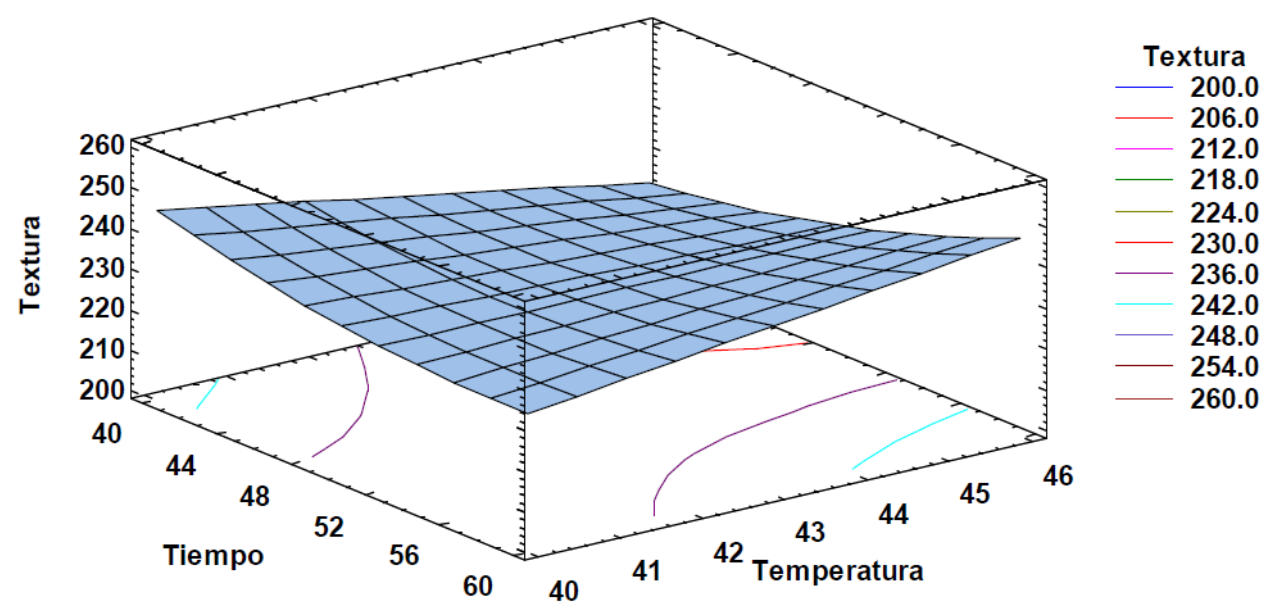

Figura 4. Superficie de respuesta de la Textura a los 0 días. 


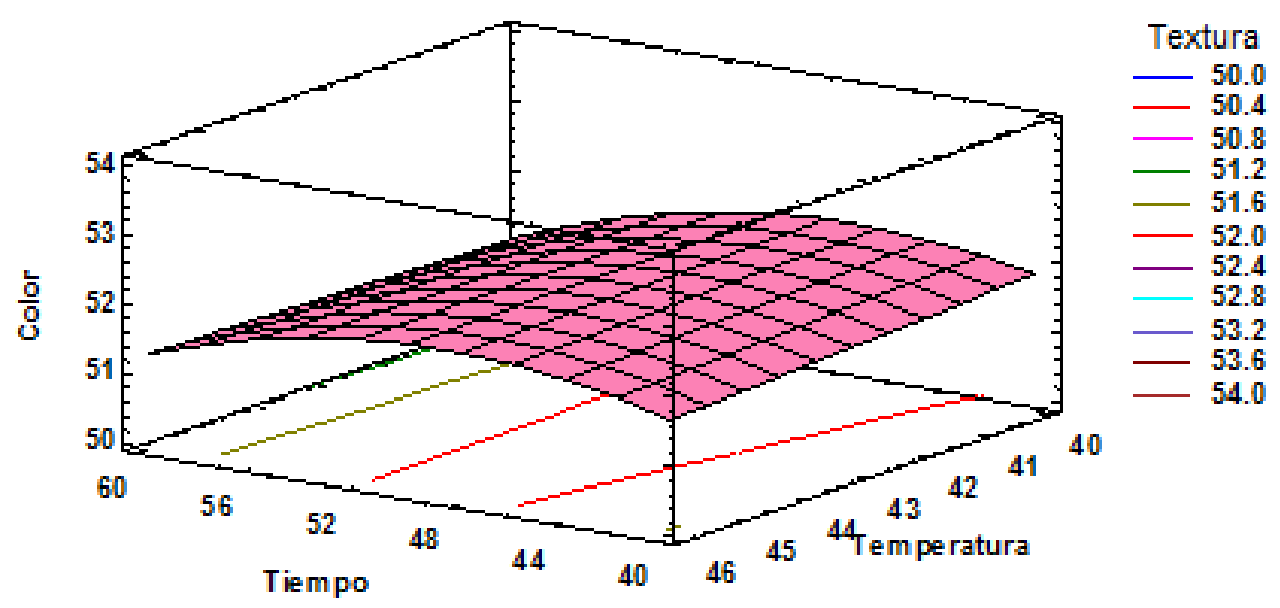

Figura 5. Superficie de respuesta del color a los 15 días.

Tabla 8. Niveles óptimos de los atributos de calidad evaluados

\begin{tabular}{lrrrr}
\hline \multicolumn{1}{c}{ Factor } & \multicolumn{1}{c}{$\mathbf{p H}$} & \multicolumn{1}{c}{ Textura } & \multicolumn{1}{c}{ Color } & \multicolumn{1}{c}{ Promedio } \\
\hline Tiempo & 53.9043 & 60.0 & 48.8726 & $54 \pm 6$ \\
Temperatura & 46.0 & 46.0 & 46.0 & 46.0 \\
Madurez & 4.0 & 4.0 & 4.0 & 4.0 \\
\hline
\end{tabular}

\section{DISCUSIÓN}

De la tabla 3, se observa la medidas biométricas de peso, longitud y diámetro ecuatorial están cercanos a los estudios (Duque et al., 2011), que se aprecian frutos mucho más grandes en cuanto a peso y tamaño en zonas ubicadas a los 2800 m n. s. m.

En cuanto al contenido de sólidos solubles, la zona de Huandobamba, se reportó $15.37^{\circ}$ Brix e Ichocan $14.50^{\circ}$ Brix, los que se ubican dentro de los estados de madurez 6 y 4 de acuerdo a las recomendaciones por INCOTEC (1999) (Vega, 2008). Al respecto, Dostert et al. (2012) indican que el fruto de la forma colombiana, es más pequeño, con un peso promedio de sólo $5 \mathrm{~g}$, con un contenido de azúcar más alto, que las otras formas y una coloración más intensa, valores cercanos a la investigación.

Respecto al porcentaje de acidez expresado en ácido cítrico, los frutos fluctúan en el rango de
2.10 (Huandobamba) a 2.14 (Ichocan), valores próximos a los reportes de INCOTEC (1999) (Vega, 2008).

El pH de los frutos se ubicaron dentro de 3.49 (Huandomba) a 3.59 (Ichocan), el cual presenta una ligera variabilidad de acuerdo a la zona de procedencia. Fueron valores cercanos a los estudios reportados por Repo de Carrasco y Encina (2008).

En cuanto al atributo de color, se obtuvieron valores en luminosidad $\mathrm{L}^{*}=62.3-66.8$ y las coordenadas de cromaticidad $\mathrm{a}^{*}=21.5$ a $21.7 \mathrm{y}$ $b^{*}=53.8-54.1$, datos cercanos a los estudios por Guevara y Málaga (2013), que reportan los rangos de color para el fruto fresco de aguaymanto de $L^{*}$ (70.31 a 71.37), a* (14.31 a 15.20) y b* (60.84 a 61.76). Al respecto Guevara y Málaga (2013), reportan $L^{*}=61.42, a^{*}=10.08$ y $b^{*}=36.52$ para un índice de madurez de 5.5. 
De la tabla 4, se observa que el aguaymanto que mantiene el cáliz durante el almacenamiento se conserva mejor, apreciaciones que coinciden Arex (citado por Ramos, 2011). Asimismo, se observa el incremento de pérdidas de peso durante el almacenamiento, incidiendo en la reducción de peso por la transpiración de las frutas y hortalizas como lo afirman Safdar et al. (Citado por Ramos, 2011).

Por otro lado, se aprecia que el aguaymanto de las zonas altas presenta mejor textura $249.18 \mathrm{gf}$ (Ichocan) en relación a las zonas bajas de 231.23 gf (Huandobamba), resultados que probablemente otorguen las condiciones climáticas de mayor resistencia en este atributo.

De la tabla 5, las medidas biométricas de peso fluctúan en los rangos de 2.60 a $4.21 \mathrm{~g}$, valores que van en aumento al cambiar de estado de madurez del 2 al 6, asociados con el desarrollo y maduración del fruto al llegar a la madurez comercial. Al respecto, Dostert et al (2012) señalan que bajo condiciones de invernadero se han registrado los siguientes valores de peso promedio del fruto: Colombia (4,2 g), Sudáfrica $(6,6$ g) y Kenia $(8,9$ g), resultados que concuerdan con el estudio.

El contenido de sólidos solubles aumentó del estado de madurez 2 al 6 de 12.20 a $14.97^{\circ}$ Brix y el porcentaje de acidez descendió de 2.33 a $1.81 \%$ de ácido cítrico e inversamente proporcional con el $\mathrm{pH}$ que fluctúa en el rango de 3.67 a 3.78, debidos al proceso de maduración de los frutos, valores que son cercanos a los reportes de INCOTEC (Citado por Vega, 2008). En cuanto a los valores obtenidos de $\mathrm{pH}$ y acidez, se encuentran dentro de los rangos mencionados por Hernández (2013), quien indica que el aguaymanto de buena calidad tiene porcentajes de acidez total titulable entre 1.6 y $2.0 \%$.

Los resultados obtenidos del color para la superficie del aguaymanto, en los estados de madurez del 2 al 6 fluctuaron para $L^{*} 66-10$ 70.9 (alta luminosidad); $a^{*} \quad 10.70-15.22$ (tonalidades rojizas) y $\mathrm{b}^{*}$ 53.40-54.89 (tonalidades amarillas), valores cercanos a los encontrados en bibliografía: $71.37 \pm 1.10 ; 15.20 \pm$ 0.48 y $61.76 \pm 1.34$, para $\mathrm{L}^{*}, \mathrm{a}^{*} \mathrm{y} \mathrm{b}^{*}$, respectivamente, reportados por Hernández (2013), que probablemente se deban al estado de madurez, ecotipo, condiciones climatológicas y prácticas culturales, entre otras (Ramos, 2011).

Los valores de textura fluctuaron de 244.5 a $156.70 \mathrm{gf}$, variaciones inherentes al estado de madurez 2 a 6, debido a los cambios en la estructura y composición de las paredes celulares, mediante la degradación e hidrolización enzimática de sustancias celulósicas, pécticas y de ácidos poligalacturónicos (Ramos, 2011).

De la prueba de preferencia entre los estados de madurez 2 y 4 , se reportó que existe suficiente evidencia estadística para aceptar que al menos uno de los estados de madurez es de diferente calidad. Para el caso de los estados de madurez 3 y 5, se registró no diferencias significativas. Sin embargo, los resultados están asociados con el menor puntaje a través de la prueba no paramétrica de Friedman, equivalente a una mayor preferencia. Asimismo estos resultados fueron contrastados mediante la prueba $t$, resultados que revelan que los estados de madurez 2 y 4 muestran diferencias significativas, reportes que coinciden con las apreciaciones de los jueces que mencionan que el estado de madurez 2, el 
aguaymanto presenta mayor acidez y de menor preferencia. Sin embargo, entre los estados de madurez 3 y 5 se aprecian no diferencias significativas, que revelan las mejores características sensoriales en el estado de madurez 5 en relación al estado de madurez 3. De éste análisis estadístico, se concluye que los estados 4 y 5 son los más preferidos, información que permitió continuar con la investigación. Al respecto, Ramos (2011) señala que los frutos desarrollan mejores características sensoriales cuanto más avanzado sea el estado de madurez en la planta madre previo a su recolección, resultados que reflejan los estudios.

De la tabla 6, los tratamientos más preferidos por los jueces se determinó el contenido de vitamina $C$ que fluctúa en el rango de 28.21 a $25.83 \mathrm{mg} / 100 \mathrm{~g}$ para los estados de madurez 4 y 5, respectivamente. Esta reducción del ácido ascórbico a medida que los frutos maduran concuerdan con los estudios de Guadarrama (Citado por Ramos, 2011), que indica que los ácidos orgánicos utilizados son empleados como sustratos respiratorios o son convertidos en azúcares.

Referente al contenido de caroteno que fluctúa en el rango 3.04 a $4.23 \mathrm{mg}$ equivalente de $\beta$ Caroteno/100g, variabilidad de acuerdo al estado de madurez que influye directamente en el contenido de compuestos bioactivos, dado que se generan durante la madurez procesos de biosíntesis los que generan mayor contenido de carotenoides, compuestos fenólicos, ácido ascórbico, etc. (Repo de Carrasco y Encina, 2008).

Como se aprecia en la figura 2, existe una variación de la textura durante el almacenamiento a los 0 días de 234.80 a 257.60 gf (aguaymanto con cáliz) y 191.30 a 229.10 gf (aguaymanto sin cáliz) y una tendencia a sufrir ablandamiento a medida que transcurre los 15 días de almacenamiento de 212.80 a $265.5 \mathrm{gf}$ (aguaymanto con cáliz) y 172.80 a 203.80 gf (aguaymanto sin cáliz), características particulares de las frutas durante el proceso de maduración. Al respecto, estadísticamente, se encontró diferencias significativas entre los bloques (p-valor <0.05). La textura a los 0 y 15 días fue menor en las muestras sin cáliz.

De acuerdo al análisis estadístico realizado (tabla 7), se observa que al inicio del almacenamiento, solo existe diferencia significativa entre los estados de madurez 4 y 5 para el pH y la textura. Además, a los 15 días, se aprecia diferencia en el color $\left(b^{*}\right)$ entre ambos estados de madurez. Finalmente, luego de 30 días de almacenamiento, no se evidencia diferencias entre los estados de madurez para ninguno de los atributos de calidad evaluados.

En comparación al testigo, a los 0 días, se observa que para el $\mathrm{pH}$ existe diferencia significativa con las muestras de estado de madurez 4. Sin embargo, no existe diferencia con las muestras en estado de madurez 5. En cuanto a la textura, a los 0 días no existe diferencia entre el testigo y el estado de madurez 4, este mejor en comparación al 5. A los 15 días, la variación del color resultó significativa. En comparación con el testigo, se observó que el mejor estado de madurez es el 5 , ya que no presenta variación con el testigo. Por tanto, el estado de madurez menos distinto en comparación al testigo sería el 5. 


\section{CONCLUSIONES}

Se concluye que la caracterización del aguaymanto en su estado de madurez comercial procedente de la zona de Ichocan reportó mejores valores promedios de peso=5.25 g, longitud=20.03 $\mathrm{mm}$, diámetro ecuatorial $=20.40 \mathrm{~mm}$, sólidos solubles $=14.50$ ${ }^{\circ}$ Brix, pH=3.59, \% acidez exp. en ácido cítrico $=2.14$, coordenadas de color $\mathrm{L}^{*}=62.3$, $\mathrm{a}^{*}=21.7 \quad \mathrm{y} \quad \mathrm{b}^{*} 54.1$ y se registraron mejor conservación las muestras de aguaymanto con cáliz.

El estudio de estados de madurez 2 al 6 recolectados de los campos de cultivo, fueron evaluados sensorialmente al cabo de 12 días de almacenamiento bajo condiciones ambientales a través de la prueba de preferencia y comparación pareada encontrando al estado de madurez 4 y 5 como los mejores.

El aguaymanto para los estados de madurez $4 \mathrm{y}$ 5 registraron un contenido de vitamina $\mathrm{C}$ de 28.21 y $25.83 \mathrm{mg} / 100 \mathrm{~g}$ y carotenoides de 3.04 y $4.23 \mathrm{mg}$ equivalente de $\beta$-Caroteno $/ 100 \mathrm{~g}$, respectivamente.

Se encontró diferencia significativa para la Textura entre las muestras sin cáliz y con cáliz y fue esta última condición considerada para continuar el estudio por ofrecer mayor estabilidad.

Durante almacenamiento, se encontró diferencias significativas para el $\mathrm{pH}$ y la Textura entre los estados de madurez 4 y 5 a los 0 días. Asimismo, a los 15 días se observó diferencia estadística para el Color $\left(b^{*}\right)$ entre ambos estados de madurez.

En función a los atributos que resultaron significativos ( $\mathrm{pH}$, Textura y Color), luego de ser analizados mediante Superficie de
Respuesta, se encontró que el tratamiento ideal que maximiza sus valores consta de: Temperatura $46^{\circ} \mathrm{C}$, Tiempo $54 \pm 6$ min y Estado de madurez 4.

Las muestras tratadas mediante el tratamiento óptimo presentó las siguientes características: sólidos solubles $15.17^{\circ} \mathrm{Brix}, \mathrm{pH}=3.95$; \% acidez exp en ácido cítrico 1.93, coordenadas de color $L^{*}=65.05, a^{*}=14.77, b^{*}=52.80$ y textura of 230.90 .

\section{Agradecimientos:}

Los autores agradecen a la Universidad Nacional Hermilio Valdizán por el financiamiento de la investigación a través de los Fondos Concursables. Al Ing. Erasmo Vásquez Rojas Gerente General de la empresa Bayas Peruanas E.I.R.L. por las facilidades con el material vegetal y al equipo de investigación de estudiantes de Ingeniería Agroindustrial-UNHEVAL por su perseverancia y apoyo incondicional.

\section{Referencias}

AOAC. (1997). Oficial Methods of Análisis. Agricultura Chemicals, Contaminants; Drugs. Vol I y II. $15^{\text {th }}$ Edición.

Dostert, N., Roque, J., Cano, A., La Torre, M. I., \& Weigend, M. (2012). Hoja botánica: Aguaymanto. Physalis peruviana L. Proyecto Perú biodiverso. Deutsche Gesellschaft für Internationale Zusammenarbeit (GIZ) GmbH. Cooperación SuizaSECO, Ministerio de Comercio Exterior y TurismoMINCETUR. Comisión de Promoción del Perú para la Exportación y el Turismo-PROMPERU. Ministerio del Ambiente-MINAM.

Duque, A. L., Giraldo, G. A., \& Quintero, V. D. (2017). Caracterización de la fruta, pulpa y concentrado de uchuva (Physalis peruviana L.). Temas Agrarios. Vol 16(1).

Guevara-Pérez, A., \& Málaga-Barreda, R. (2013). Determinación de los parámetros de proceso y 
caracterización del puré de aguaymanto. Ingeniería Industrial, (031), 167-195.

Hernández, C. C. (2013). Desarrollo de productos tratados por procesos térmicos y no térmicos a partir del fruto physalis peruviana linnaeus. Memoria para optar al título de ingeniera en alimentos. Universidad de Chile. Facultad de Ciencias Químicas y Farmceúticas. Chile.

Quintero, G.A., Castro, K. \& Taborda, G. (2012). Evaluación sensorial de Uchuva Physalis peruviana, L.) proveniente de tres regiones de Colombia. Revista de la asociación colombiana de ciencia y tecnología, 21(26), 18-25.

Ramos, M. (2011). Efecto del tratamiento hidrotérmico en la conservación postcosecha del tomate (Solanum lycopersicum, L.) cv. Nabateo. Tesis paraoptar el Grado de Magister Scientiae en Tecnología de Alimentos. Universidad Nacional Agraria La Molina.

Ramos, M. 2013.Guía de prácticas de control total de calidad, dirigido a los estudiantes de la EPIA de la Universidad Nacional Hemilio Valdizan, Huánuco, Perú.
Repo de Carrasco, R., \& Encina Zelada, C. R. (2008). Determinación de la capacidad antioxidante y compuestos bioactivos de frutas nativas peruanas. Revista de la sociedad química del Perú, 74(2), 108-124.

Rolle, L., Siret, R., Segade, S. R., Maury, C., Gerbi, V., \& Jourjon, F. (2012). Instrumental texture analysis parameters as markers of table-grape and winegrape quality: A review. American Journal of Enology and Viticulture, 63(1), 11-28.

Rolz, C. (2011). Fisiología poscosecha de frutas. Compendio de características de calidad, condiciones de almacenamiento, sensibilidad al frio, maduración y desórdenes fisiológicos. Rev. Univ. Val. Guatem, 23, 23-34.

Talcott y Howard. (1999). Determinación de carotenoides totales.

Vega, N. (2008). Normas internacionales en la exportación de uchuvas desde el puerto de Santa Marta. Universidad Sergio Arboleda Santa Marta. 